\title{
Platin-Auszeichnung für Charité
}

Am 21.08.2020 hat die Klinik für Radiologie der Charité als erste deutsche Klinik im Rahmen des European Training Assessment Programme ${ }^{1}$ (ETAP) des European Board of Radiology (EBR) das Platin-Zertifikat erlangt. Mit dieser Zertifizierung werden Weiterbildungsstätten für Radiologie in Europa akkreditiert.

Mit der Platin-Auszeichnung - als höchstmöglichem Zertifikat - akkreditierte Kliniken zeichnen sich unter anderem aus durch

- die Verfügbarkeit von spezialisiertem Personal für die Weiterbildung der Assistenzärzt*innen,

- ausgezeichneter und persönlicher Supervision der Weiterbildungsassistent*innen,

- einem Mentor*innenprogramm für die Karriereentwicklung des Nachwuchses,

- die spezifische und frühzeitige Weiterbildung in der Notfallradiologie,

1 EBR. How to apply for an ETAP assessment. 2020 https://www.myebr.org/etap/ apply-etap-assessment. Zuletzt abgerufen 23.09.2020.
- die Einbindung der Weiterbildungsassistent*innen in interdisziplinären Klinikbesprechungen,

- der Möglichkeit in Wissenschaft, Management und Führungsaufgaben tätig zu werden sowie

- eine Ausrichtung nach dem europäischen Weiterbildungs-Curriculum.

Die Charité als gemeinsames Universitätsklinikum der Freien Universität Berlin und der Humboldt-Universität zu Berlin versorgt jährlich ca. 700000 ambulante und 150000 stationäre Fälle. Die radiologische Patientenversorgung umfasst mit ca. 1700 Untersuchungen proTag die Betreuung der stationären Patienten, die ambulante radiologische Versorgung der eigenen Ambulanzen und externer Zuweiser sowie die Befundung der teleradiologisch übermittelten Bildgebung von aktuell 12 Kliniken. Die Klinik für Radiologie betreut die 3 Campi der Charité, Charité Campus Benjamin Franklin (CBF), Charité Campus Mitte (CCM) und Charité Campus Virchow-Klinikum (CVK). Die Weiterbildung von insgesamt 74 Assistenzärzt*innen wird durch 62 Fach- und Oberärzt*innen gewährleistet, die das gesamte Spektrum der
Arbeitsgemeinschaften der Deutschen Röntgengesellschaft ${ }^{2}$ (DRG) und Subspezialisierungen der European Society of Radiology ${ }^{2}$ (ESR) abdecken und so eine Weiterbildung in höchster Qualität ermöglichen.

Die Klinik für Radiologie ist abgesehen von der Mammabildgebung und der interventionellen Radiologie vorwiegend modalitätenorientiert strukturiert. Die ersten Weiterbildungsjahre vermitteln Basiswissen, -fertigkeiten und -kompetenzen in den Modalitäten der konventionellen Projektionsradiografie, Computertomografie und Sonografie, die für die Bereitschaftsdienste vorausgesetzt werden. Dienste werden nach umfangreicher Weiterbildung in der Akutdiagnostik von Weiterbildungsassistent*innen in der Regel ab dem zweiten Jahr besetzt. Mit steigender Erfahrung werden ebenfalls klinisch-radiologische Falldemonstrationen durchgeführt. Um einen umfassenden Einblick in alle Gebiete der radiologischen Bildgebung innerhalb der

2 Deutsche Röntgengesellschaft. Arbeitsgemeinschaften in der DRG. 2020 https://www. drg.de/de-DE/14/arbeitsgemeinschaften/. Zuletzt abgerufen 23.09.2020. 
Weiterbildungszeit zu erhalten, werden die Falldemonstrationen organbasiert in Teams (Mammabildgebung, kardiale und vaskuläre Bildgebung, Thoraxbildgebung, Intensiv- und Notfalldiagnostik, Muskuloskelettale Bildgebung, gastrointestinale und abdominelle Bildgebung, Kopf-Hals-Bildgebung, Hämato-onkologische Bildgebung, Urogenitale Bildgebung) koordiniert und 6-monatliche Rotationen in diesen Teams unabhängig vom Status der modalitätenbasierten Rotation in der Weiterbildung angeboten. Nach den grundlegenden Rotationen folgen die Modalitäten MRT, Mammografie, Durchleuchtung und interventionelle Radiologie.

Neben der radiologischen und interventionellradiologischen Weiterbildung erfolgen auch Rotationen in die Neuroradiologie und ab dem Facharzt ggf. Weiterbildungsoptionen in Nuklearmedizin und Kinderradiologie.
Die Supervision erfolgt in der Klinik für Radiologie direkt und fallbasiert. In jeder Modalität (und jedem Campus) steht mindestens ein/e supervidierende/r Fachärzt*in zur Verfügung. Die Evaluation der persönlichen Leistung wird in persönlichen Gesprächen am Ende jeder Rotation und in einem Jahresgespräch mit dem Klinikleiter durchgeführt.

Die Mitarbeiter*innen der Klinik für Radiologie zeichnen sich durch ein hohes Engagement in der Lehre und in der Forschung aus (so konnten sich seit 1994 mehr als 60 Kollegen*innen für das Fachgebiet der Radiologie habilitieren).

Neben der Klinik für Radiologie der Charité haben bisher die radiologischen Kliniken des King's College Hospital in London und des Universitätsspitals Basel diese Zertifizierungsstufe erreicht ${ }^{3}$.
Die Klinik für Radiologie der Charité - Universitätsmedizin Berlin unterstützt ausdrücklich das Engagement des EBR, die Qualität von Lehre und Weiterbildung abzubilden und so eine Förderung der Qualität in der Patientenversorgung zu bewirken. Bei Interesse zum Erfahrungsaustausch über die Strukturierung der Weiterbildung und dem Ablauf des Zertifizierungsprozesses stehen die Autoren gern zur Verfügung.

Frau Anne Frisch, Dr. Julian Pohlan, Dr. LarsArne Schaafs Prof. Dr. Bernd Hamm, Prof. Dr. Marc Dewey
32020 https://www.myebr.org/etap/accreditedcentres. Zuletzt abgerufen 23.09.2020. 